\title{
Monitoring aerosols over Europe: an assessment of the potential benefit of assimilating the VIS04 measurements from the future MTG/FCI geostationary imager
}

Maxence Descheemaecker ${ }^{1}$, Matthieu Plu ${ }^{1}$, Virginie Marécal ${ }^{1}$, Marine Claeyman ${ }^{2}$, Francis Olivier ${ }^{2}$, 5 Youva Aoun ${ }^{3}$, Philippe Blanc ${ }^{3}$, Lucien Wald ${ }^{3}$, Jonathan Guth ${ }^{1}$, Bojan Sič ${ }^{1}$, Jérôme Vidot ${ }^{4}$, Andrea Piacentini $^{5}$, Béatrice Josse ${ }^{1}$

1CNRM, Météo-France - CNRS, UMR3589, 31057, Toulouse, France

2Thales Alenia Space, Cannes la Bocca, 06156, France

10 3Mines ParisTech, PSL Research University, O.I.E.- Center for Observation, Impacts, Energy, Sophia Antipolis, 06904, France

4Centre de Météorologie Spatiale, Météo-France, 22300, Lannion, France

5CERFACS - CNRS, UMR5318, 31057, Toulouse, France

Correspondence to: Maxence Descheemaecker (maxence.descheemaecker@ meteo.fr)

151 Supplementary Material 

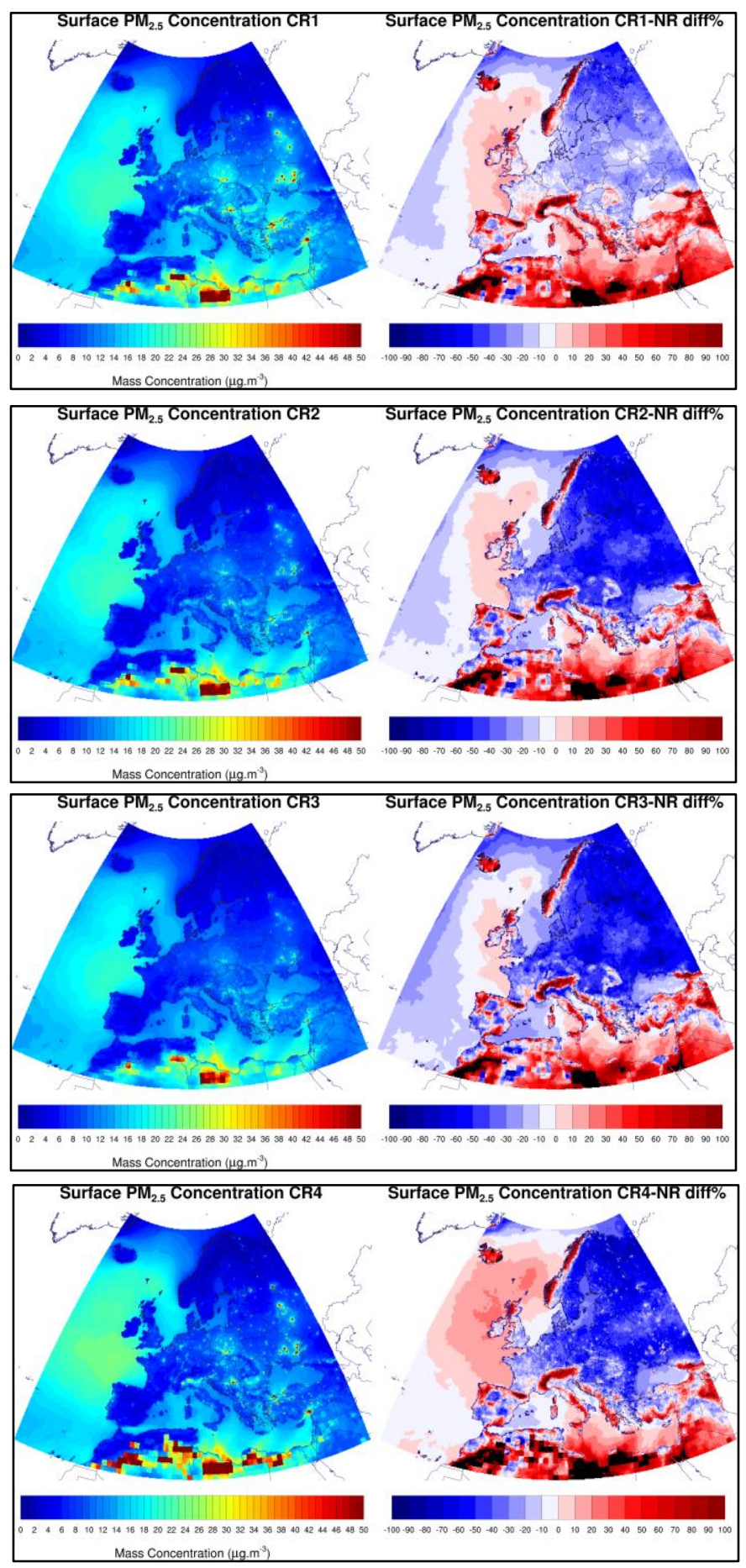

Figure S1: For each CR (CR1, CR2, CR3 and CR4), the figures represent a PM2.5 comparison between the NR and the CRs from January to April 2014: the mean surface concentration of the CR and the normalized differences between CRs and the NR. 

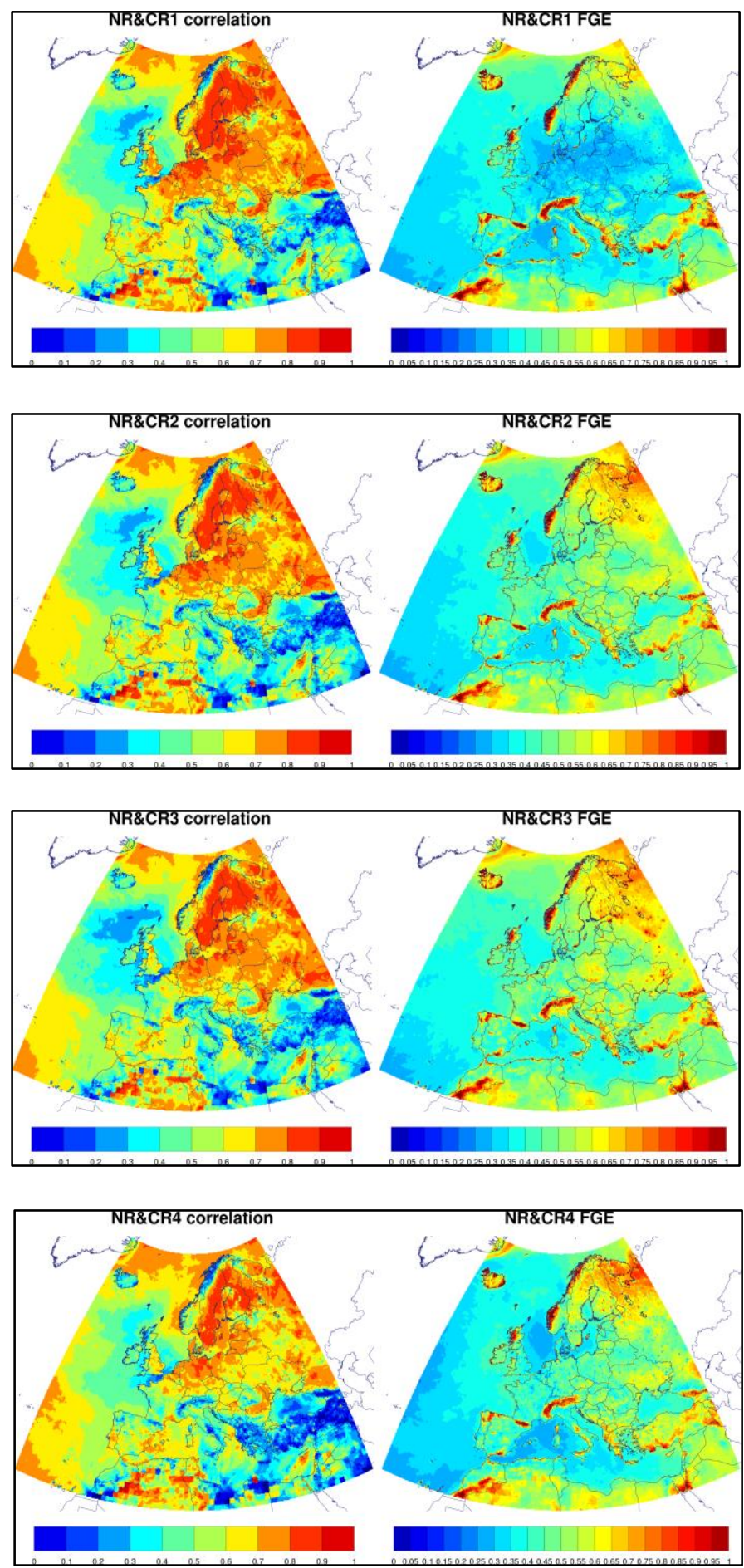

Figure S2: For each CR (CR1, CR2, CR3 and CR4), the figures represent a PM 2.5 comparison between the NR and the CRs from January to April 2014: the Pearson correlation and the fractional gross error. 

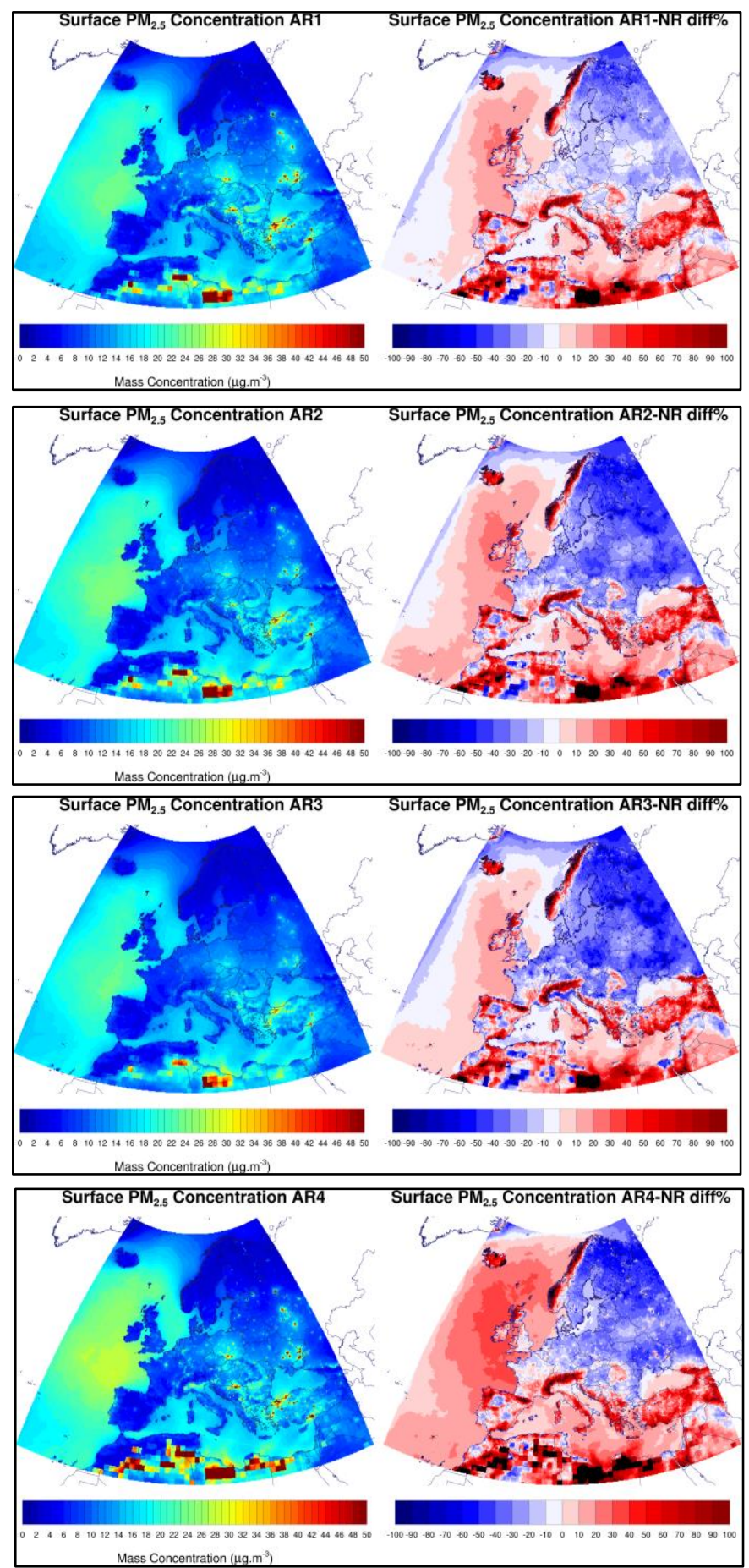

Figure S3: For each AR (AR1, AR2, AR3 and AR4), the figures represent a PM2.5 comparison between the NR and the ARs from January to April 2014: the mean surface concentration of the $A R$ and the normalized differences between ARs and the NR. 

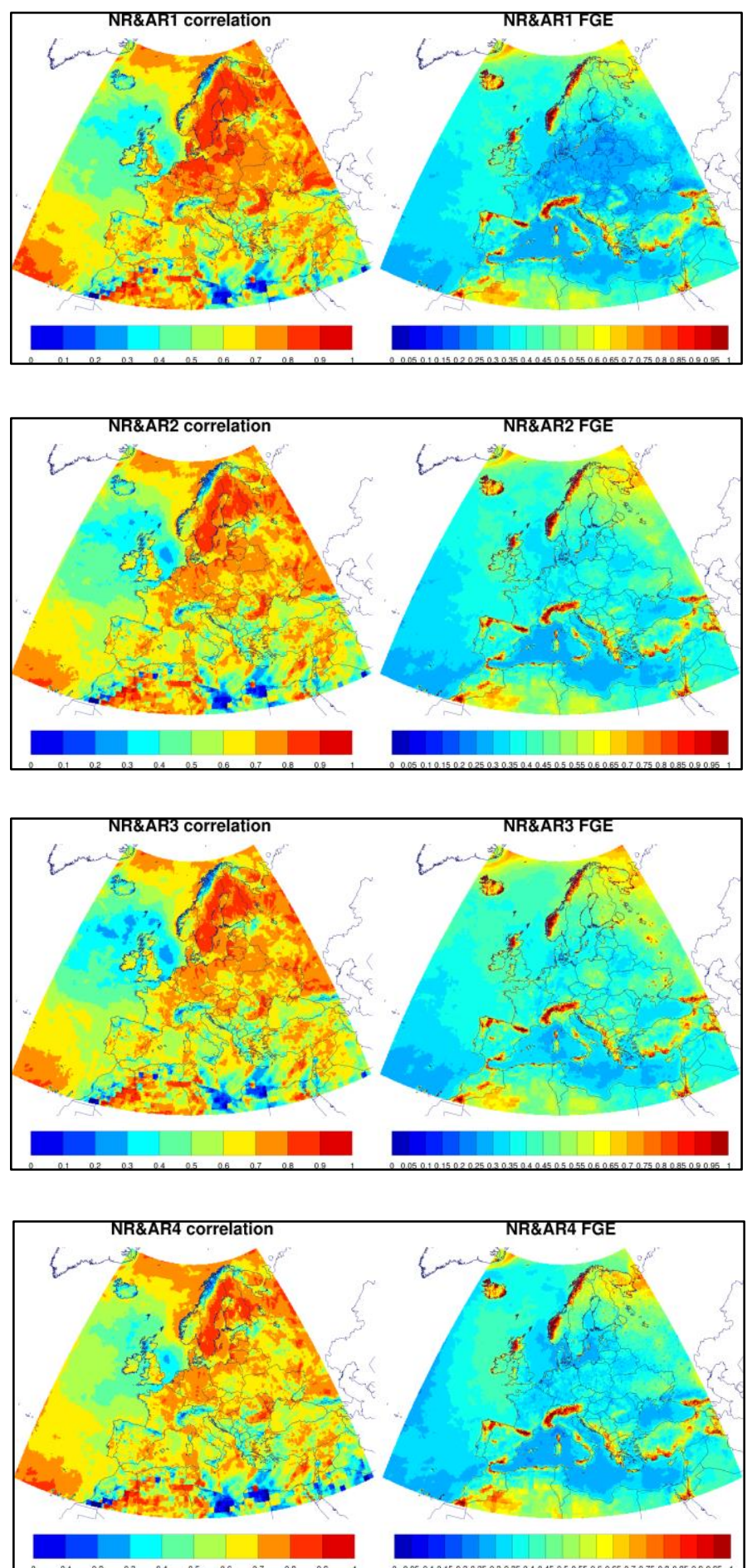

Figure S4: For each AR (AR1, AR2, AR3 and AR4), the figures represent a $\mathrm{PM}_{2.5}$ comparison between the NR and the ARs from January to April 2014: the Pearson correlation and the fractional gross error. 

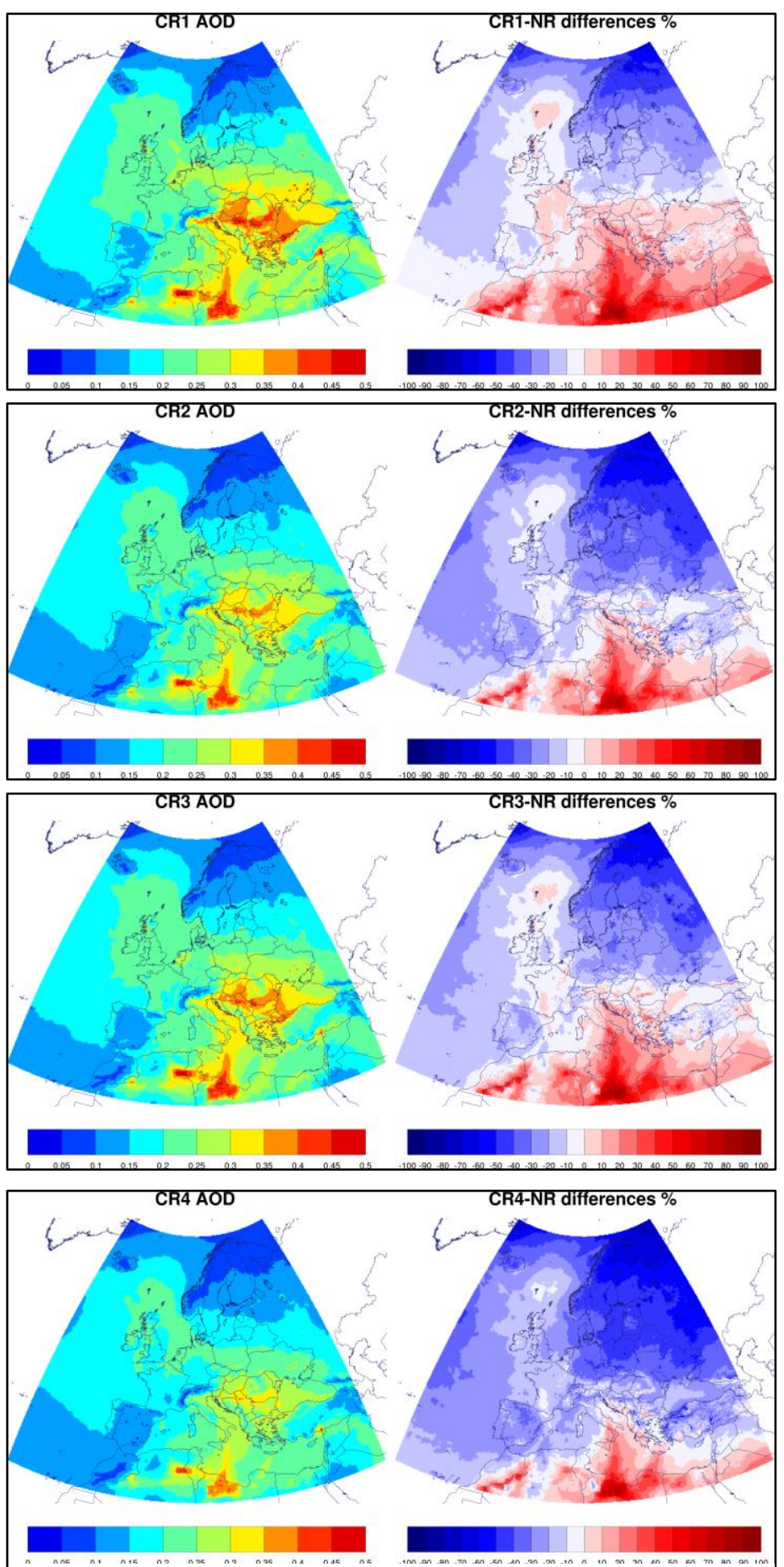

Figure S5: For each CR, the figures represent the mean AOD of the CR and the normalized differences between the NR and the CR for the period from January to April 2014. 


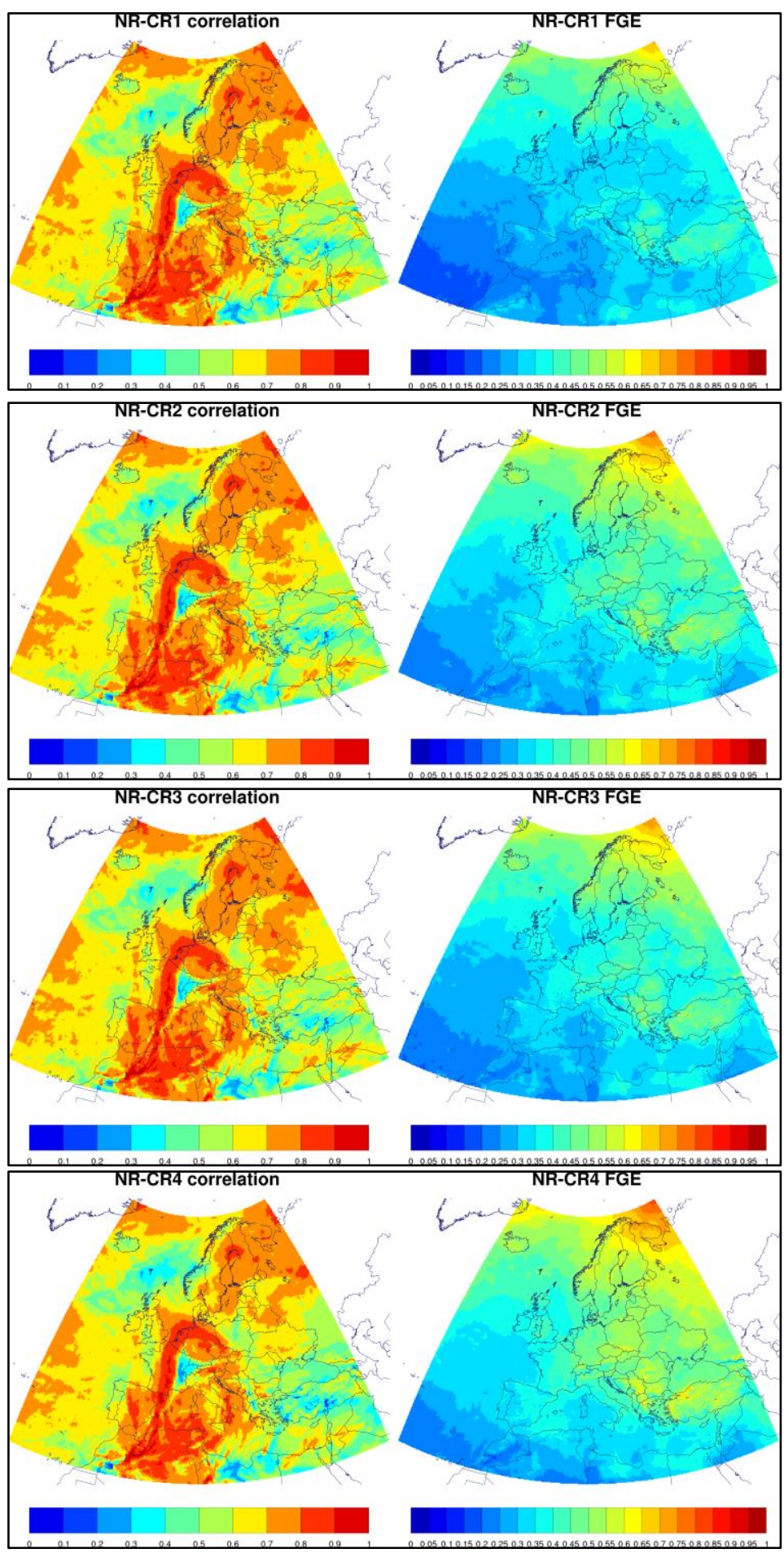

Figure S6: For each CR, the figures represent the Pearson correlation and the fractional gross error of AOD between the NR and the CR for the period from January to April 2014. 

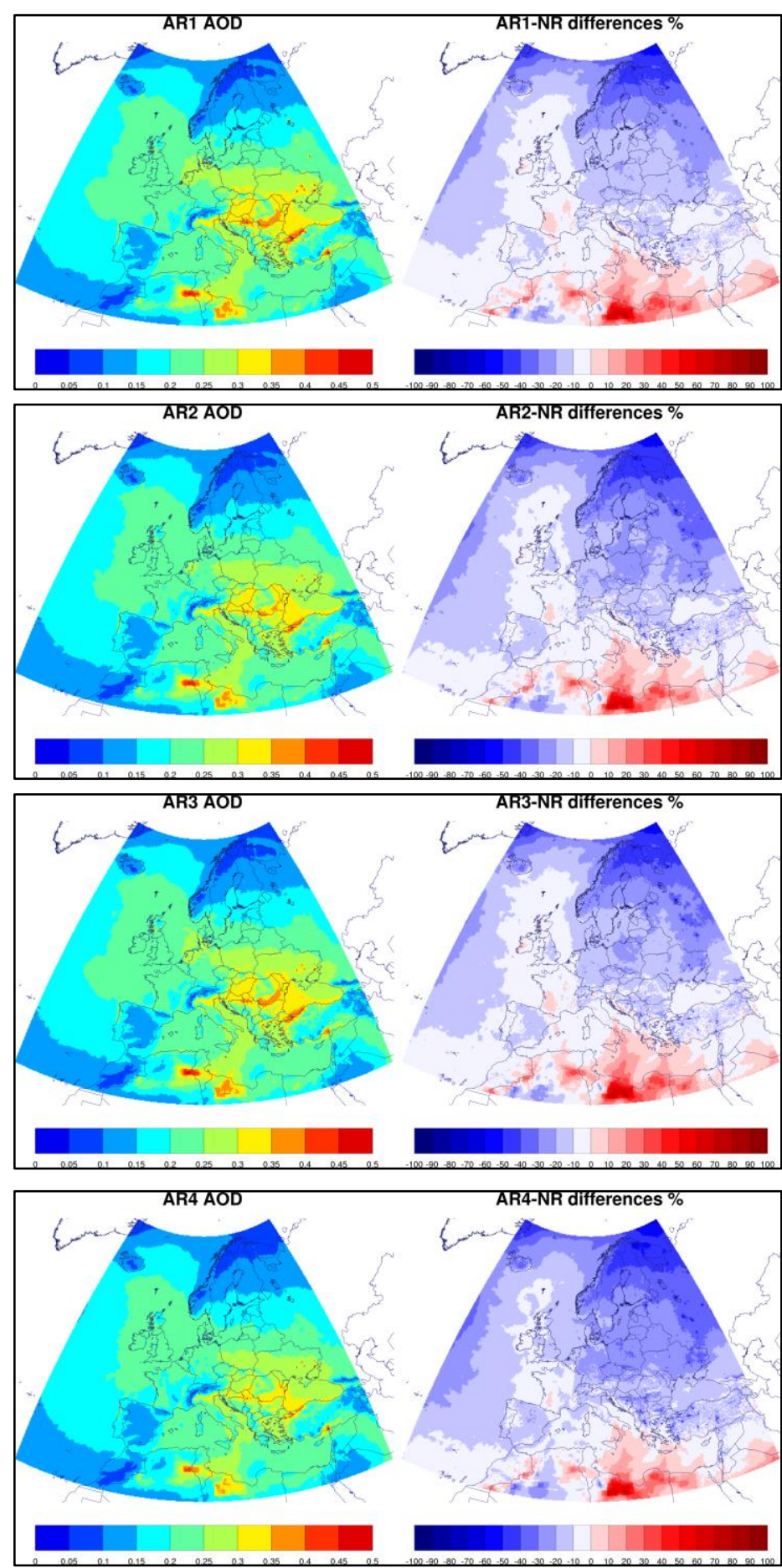

Figure S7: For each AR, the figures represent the mean AOD of the AR and the normalized differences between the NR and the AR for the period from January to April 2014. 

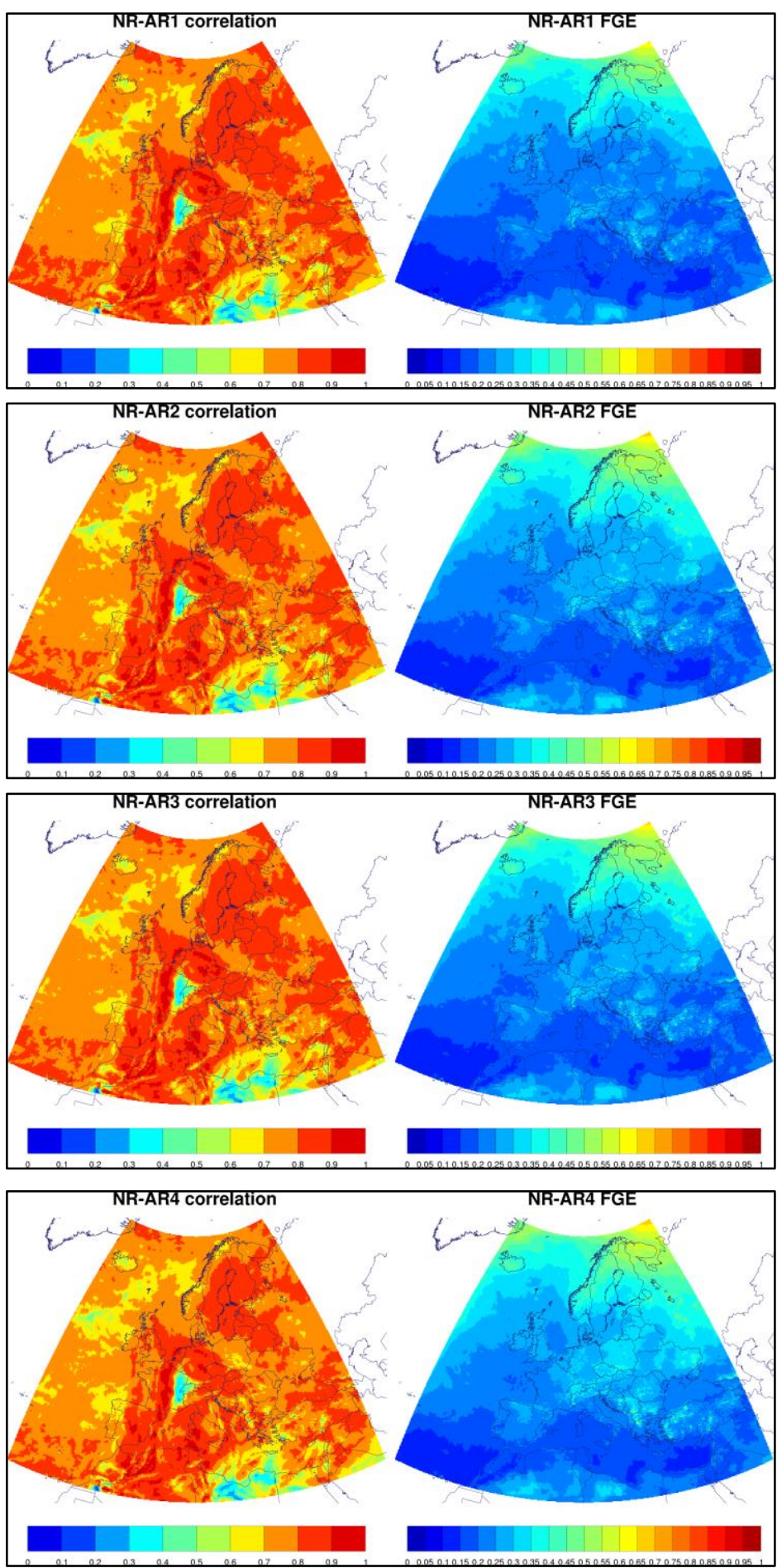

Figure S8: For each AR, the figures represent the Pearson correlation and the fractional gross error of AOD between the NR and the AR for the period from January to April 2014. 